\title{
Evaluation of Stimulation Threshold in Nerve Cells by Exposure to Time-varying Magnetic Field in In Vitro
}

\author{
Masateru IKEHATA \\ Biotechnology Laboratory, \\ Human Science Division \\ Atsushi SAITO \\ Central Research Institute \\ of Electric Power Industry
}

\author{
Yukihisa SUZUKI \\ Tokyo Metropolitan University
}

\author{
Sachiko YOSHIE \\ Biotechnology Laboratory, \\ Human Science Division
}

\begin{abstract}
International guidelines that aim to protect people from adverse health effects of exposure to magnetic fields (MF) have been established by international organizations such as the International Commission on Non-Ionizing Radiation Protection (ICNIRP) or the Institute of Electrical and Electronic Engineers (IEEE). In these guidelines, one of the target effects below $100 \mathrm{kHz}$ is nerve stimulation based on a few perceptual studies. Because of a few studies, the value of basic restriction of the guidelines adopt extrapolation widely such as $45 \mathrm{~Hz}$ to $400 \mathrm{~Hz}$ and $7 \mathrm{kHz}$ to 100 $\mathrm{kHz}$. Therefore, determination of threshold value among extrapolated frequencies is important for verification and improving of the current basic restriction. Here, we report progress of our study to determine threshold value of nerve stimulation by magnetic or electric field in in vitro.
\end{abstract}

Keywords: magnetic field, ICNIRP guidelines, health effect of magnetic fields, nerve stimulation, basic restriction, reference level

\section{Introduction}

It has been 40 years since the discussion on the health risks of electromagnetic fields began, starting with the results of an epidemiological study of residents in the vicinity of transmission lines in the United States [1]. Various initiatives have been carried out since then, and management of health risks related to magnetic fields on railways in Japan was also introduced in 2012 with the revision of the Ordinance for Technical Regulatory Standards on Japanese Railways, which provides regulations governing the location of fixed electrical railway installations in way that prevents any impact on the health of the general public in the vicinity from power frequency magnetic fields $(50 / 60 \mathrm{~Hz})$.

This regulation is based on a guideline released by the International Commission on Non-Ionizing Radiation Protection (ICNIRP) and applies only to power frequency magnetic fields from fixed electrical installations for railways.

However, future changes need to be taken into account, such as:

- Expansion of magnetic field regulations (elimination of specific frequencies);

- Increase of magnetic fields in rail cars due to increased power consumption to achieve higher speeds.

Since these changes may generate issues relating to the safety of railways in the future.

In addition, in the ICNIRP guidelines, "transient nerve stimulus effect by magnetic field exposure" should be prevented, however, in the setting of basic restrictions, there are frequency bands which are extrapolated and interpolated with experimental and theoretical studies.

In the context of magnetic fields in railway systems, the purpose of this study was to obtain biological data relating to the frequency bands to be considered for use in the railways, including extrapolated bands in the ICNIRP guideline.
2. International guidelines to protect people from the adverse effects of magnetic field exposure

The regulation of magnetic fields in railway systems is based on "GUIDELINES FOR LIMITING EXPOSURE TO TIME-VARYING ELECTRIC AND MAGNETIC FIELDS (1 $\mathrm{Hz}$ TO $100 \mathrm{kHz}$ )" revised by the ICNIRP in 2010 [2].

These guidelines are based on the WHO's Environmental Health Criteria [3], which aim to protect the human body only from short-term and acute effects (nerve stimulation, etc.) of exposure to strong magnetic fields, and do not include long-term and chronic effects, such as correlation between long-term weak magnetic field exposure and the risk of developing childhood leukemia.

In the guideline, amount of electric field induced by the magnetic field is targeted as the physical quantity to be protected, and the exposure limits are defined as "basic restrictions" (Fig. 1) based on the research of volunteers on magnetic flash $(10 \mathrm{~Hz}$ to $45 \mathrm{~Hz}$ ) and peripheral nerve perception $(500 \mathrm{~Hz}$ to $7 \mathrm{kHz}$ ), including one frequency band (45 Hz to $400 \mathrm{~Hz}, 7$ $\mathrm{kHz}$ to $100 \mathrm{k} \mathrm{Hz}$ ) extrapolated without experimental evidence.

Electromagnetic fields in railway systems (railway magnetic fields) are characterized by the simultaneous generation of multiple frequencies from different facilities, along with power frequency magnetic fields in fixed electrical installations, static magnetic fields associated with direct current supply, or time-varying magnetic fields in which frequencies and intensity change from the conversion devices or reactor devices to drive trains. Moreover, the strength of magnetic fields in the railway system is relatively high compared to power lines and other equipment used in electrical utilities, because there are more situations where people may be close to electrical equipment, although levels are low enough to fall within the threshold values in the guidelines. 


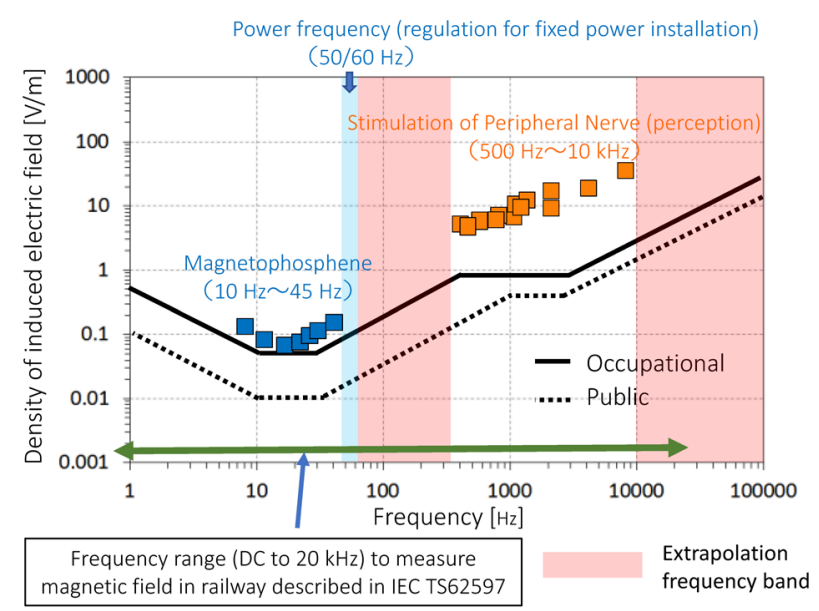

Fig. 1 Basic restriction of the ICNIRP guideline and its extrapolation frequency bands $\square$ and $\square$ show thresholds of previous studies. shows extrapolation frequency bands due to lack of scientific evidence.

Therefore, the purpose of this study is to clarify experimentally the frequency characteristics of neural stimulation including the extrapolated frequency band in the ICNIRP guidelines which may be generated as railway magnetic fields. We focused on cultured neuron cells obtained from the brain of experimental rat fetuses instead of human cells, or experimental animals, because it is ethically difficult to study experimental stimulus responses in humans and experimental animals. On the other hand, it is possible to increase the number of experiments and study a wide range of experimental conditions using cultured cells, because a sufficient amount of homogeneous cells can be obtained and it is easy to control the experimental environment. In this paper, we report the findings on the threshold of the stimulus response in neuron cells due to magnetic/ electric field exposure using unique experimental systems. These findings form part of the biological evidence for the extrapolated frequency band in the ICNIRP guidelines.

\section{Primary nerve cell culture of rat fetus}

Since the cells of a particular part of an individual are considered to retain the function of tissue in vitro, it is considered to be highly valuable as an experimental system that reproduces tissue function, and is very effective for evaluation of various effects. Therefore, we decided to use primary cultured cells. We used nervous system cells that were derived from the cerebral cortex of a rat fetus' brain. The cell suspension was prepared from the cerebral cortex of a fetus brain, then cultured in Neurobasal medium supplemented with $2 \%$ B-27 supplement (Thermo Fisher Co., Ltd.) and antibiotics located in $5 \% \mathrm{CO}_{2}$ with saturated water vapor at $37^{\circ} \mathrm{C}$.

During the culture, the appearance of neurite outgrowth was observed in a couple of days (Fig. 2). The cells were stabilized after 14 days and spontaneous neuronal activities were clearly observed as a change in their fluorescence intensity labelled by Fluo-4-AM, a calcium ions indicator. Using this primary cell culture, the stimulus response thresholds by exposure to magnetic field or external electric field were examined.

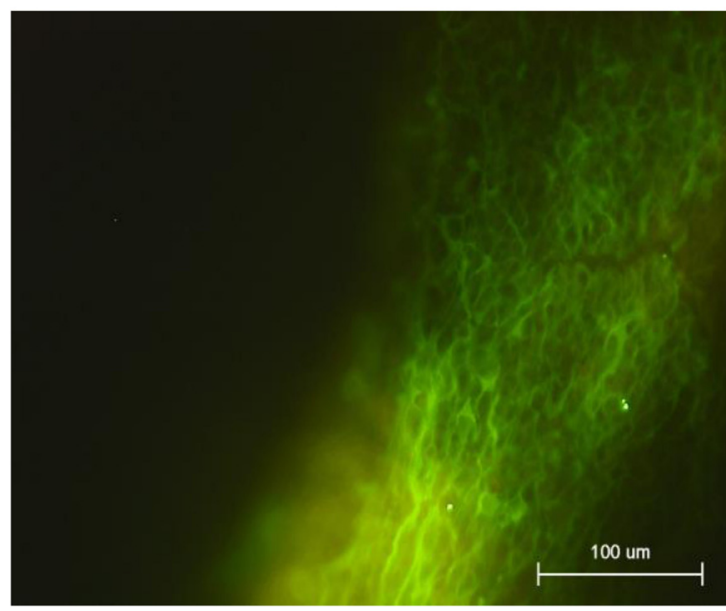

Fig. 2 Typical microscopic observation of primary nerve cell culture of cerebral cortex of rat fetus Cells were immunohistochemically stained by antimap2 antibody labeled with alexa-488.

\section{Observation of threshold of nerve stimulation}

\subsection{Stimulation of nerve cells by time-varying mag- netic field}

ICNIRP guidelines, which provide reference values for magnetic field regulation in the railways, set a basic limit on the amount (basic restriction) of induced electric fields within the body by time-varying magnetic fields. However, since it is difficult to measure the induced electric field within the body, the magnetic field strength to induce the level of electric field in the basic restriction is estimated by numerical calculation using voxel human model as an index that can be easily evaluated, and is called the "reference level". As a result, measurement of magnetic fields in a targeted space is used for compliance evaluation.

Since the induced electric field within the body by magnetic fields increases as a concentric shape depending on the area across which the magnetic flux crosses, the larger the area, the stronger the induced electric field of the outer periphery. Since the reference level in the ICNIRP guidelines is based on human dimensions, it is necessary to set experimental conditions based on extent of the induced electric field rather than strength of magnetic field. Generally, since culture dishes for cells are small compared to humans, a stronger magnetic field is required to obtain the same level of induced electric field. In addition, as a characteristic of the stimulation response of a living body, the threshold of the induced electric field for nerve stimulation increases with the frequency of magnetic field due to the electrical properties of the cell membrane [4, 5]. Therefore, in order to obtain a threshold with a higher frequency in the cell experiment system, a stronger magnetic field is required, which generates significant cost because of the need to construct a magnetic field generator.

Therefore, we focused on external electric fields, which could be applied using a U-shaped electrode located in the vicinity of the cell. This made it possible to examine the threshold of a wide frequency range of external electric fields compared with exposure to time-varying magnetic fields. 


\subsubsection{Design of a real-time single cell observation system during nerve cell stimulation by strong time-varying magnetic field}

The basic restrictions in the ICNIRP guidelines described in Chapter 2 were mainly established on the basis of perceptual experiments of volunteers, and the extent of induced electric fields at these thresholds form the reference for in vitro cell experiments. Therefore, we targeted $4 \mathrm{~V} / \mathrm{m}$, which is the stimulation threshold of peripheral nerves, and examined the magnetic flux density that induced the level of electric fields in a culture medium. When the conductivity of a typical medium (D-MEM) was approximately $1.7 \mathrm{~S} / \mathrm{m}$, the required magnetic flux density was estimated to be inversely proportional to frequency, approximately $2000 \mathrm{MT}$ or more at $50 \mathrm{~Hz}, 1000 \mathrm{mT}$ or more at $100 \mathrm{~Hz}$, and $500 \mathrm{mT}$ or more at $200 \mathrm{~Hz}$. Figure 3 shows the estimated results of the induced electric field at 200 $\mathrm{Hz}$. When the coil is modeled and the position of the cells using a culture dish of $90 \mathrm{~mm}$ diameter was set to $10 \mathrm{~mm}$ above the coil, exposure of $500 \mathrm{mT}$ reveals an induced electric field of 4 to $5 \mathrm{~V} / \mathrm{m}$ in a region 30 to $50 \mathrm{~mm}$ horizontally away from the center of the coil. On the other hand, the coil that generates this magnetic flux density on the culture surface of the cell, the amount of current that can flow to the available wire and its wire, such as heat generation and electromagnetic force associated there with it, there are constraints on realistic production, thus there is a limit to generate magnetic flux density. Under these constraints, we examined and optimized the design of the coil and the estimation of the induced electric field at the bottom of the culture dish by the magnetic field generated from the coil, and in this study, we designed and manufactured a coil and power unit to generate a maximum of $500 \mathrm{mT}$ or more at the center of the culture dish bottom $(10 \mathrm{~mm}$ above the coil surface) in $200 \mathrm{~Hz}$. As a result, the spiral coil which was the most advantageous to generate strong magnetic field which has three identical layer of 25 turns was created. This coil can generate a magnetic flux of $500 \mathrm{mT}$ when driven at a current of $200 \mathrm{~Hz}, 500 \mathrm{~A}$. The power unit for driving the magnetic field-generated coil was designed and manufactured to allow 10 consecutive sine wave currents to flow in a single exposure, taking into account the heat generation of the coil (Fig. 4) [6].

Commonly used metals like platinum electrodes which measure the response of nerve cells cannot be used in environments where cells are exposed to strong time-varying magnetic fields due to heat and noise generation. Therefore, we used a method for tracing intracellular calcium

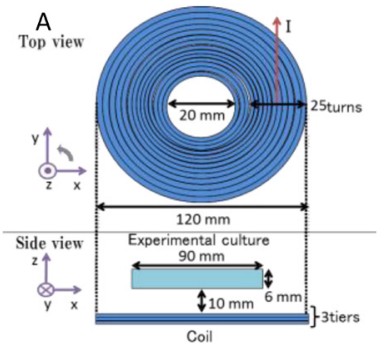

B

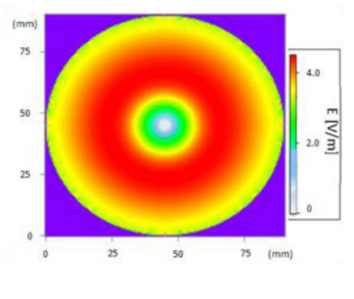

Fig. 3 Estimation of induced electric field A: Modeling of exposure coil, B: Dosimetry of experimental culture

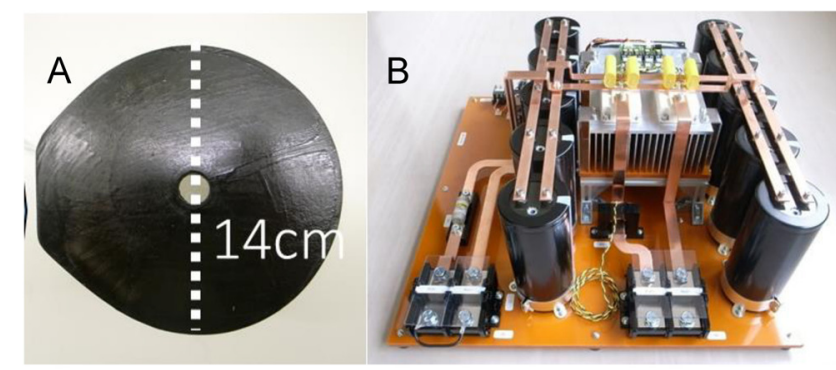

Fig. 4 Electromagnetic coil (A) and Power unit (B) for generation of strong time-varying magnetic field

ions which fluctuate greatly during nerve cell responses by labeling them with a fluorescent dye and tracing them. An inverted microscope is normally used to visualize the dish in which the cells are cultured from below, but the exposure coil is placed immediately below the cell dish, so observations should ideally be done from above and from a good working distance. We therefore used a macro zoomlens microscope system (Keyence Co., Japan). We designed and created a dedicated resin stand which retained the optical fibers of the light source, microscope, magnetic fieldgenerating coil, and the sample container in the laboratory to generate the magnetic field. The stands for the exposure coil and sample were spatially separated and stand-induced vibrations during magnetic field generation were prevented. A high-sensitivity cooled CCD camera (Hamamatsu Photonics Co., Japan) which was connected to the upper end of the lens and a control / analysis software (MV-DOC, Molecular Devices, LLC) were used to obtain microscopic images of samples containing the excited fluorescence.

Fluo-4-AM (Dojindo Laboratories, Japan), which is fluorescent dye that binds with calcium ions, was used for observations of intracellular calcium ions. Cells were left standing in a buffer which contained surfactant to allow dye to be intracellularly incorporated, after which the dye-incorporated cells were left to stand directly below a microscopic lens inside a temperature-adjustable cell culture container (Blast, Co., Ltd., Japan) composed of ITOtransparent electrodes. The temperature of the cell culture container was adjusted so that the thermometer in contact with the dish recorded a temperature of $37 \pm 1^{\circ} \mathrm{C}$. The fluorescent dye incorporated in the cell was then excited with blue light $(488 \mathrm{~nm}$ ) from the fluorescent light source, and the amount of green fluorescence which depended on the amount of intracellular calcium ions were imaged and recorded at an exposure time of 0.5 seconds using a high-sensitivity cooled CCD camera. The overall structure observation device of the time-varying magnetic field stimulation is shown in Fig. 5.

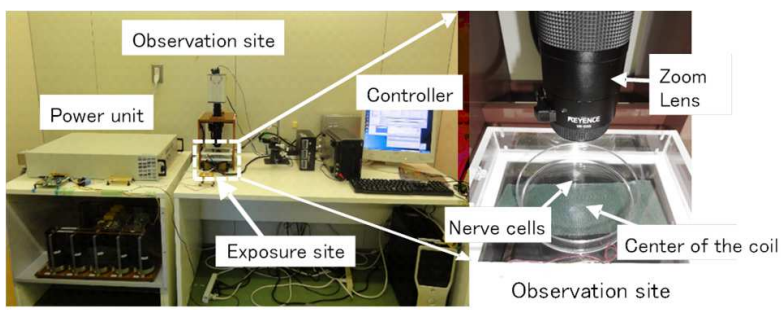

Fig. 5 The entire structure of the magnetic field Stimulation observation system 

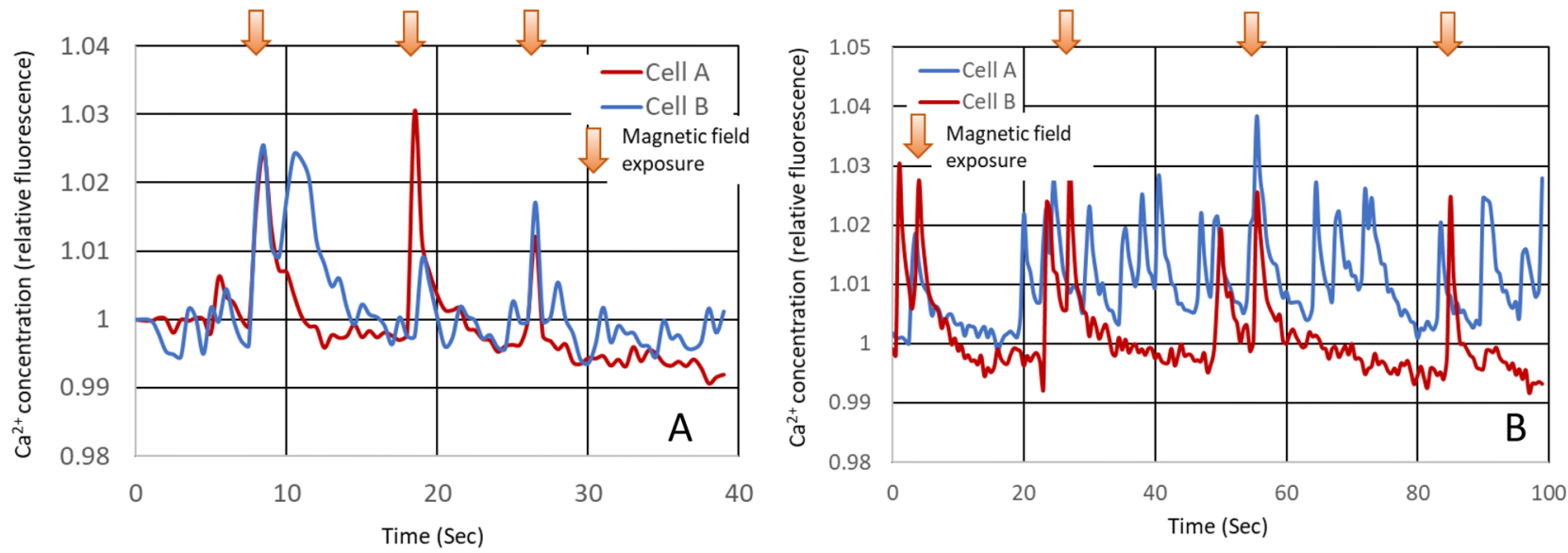

Fig. 6 Typical experimental results of magnetic stimulation of cultured rat nerve cells A: Exposure to $200 \mathrm{~Hz}, 750 \mathrm{mT}$, B: Exposure to $400 \mathrm{~Hz}, 500 \mathrm{mT}$

\subsubsection{Observation of stimulation due to the time- varying magnetic field exposure}

The amount of induced electric field due to the timevarying magnetic field increases as the magnetic flux density and the cross-sectional area of the flux increase. Therefore, we selected the maximum culture container which fits in the observed section (square dish $(86 \times 128 \mathrm{~mm})$ ) a nerve cell-cultured cover glass was set up in the $25-30-\mathrm{mm}$ area from the center of the dish where the induction amount was thought to be maximal after the buffer solution for observations was removed in order to increase the amount of induction into the cells as much as possible. The magnetic field exposure conditions were set at a maximum of $200 \mathrm{~Hz}$ and $750 \mathrm{mT}$.

Typical result of calcium ion dynamics according to the observed cells are shown in Fig. 6. Periodic spontaneous activity of the cells themselves could be observed in a $200 \mathrm{~Hz}, 750 \mathrm{mT}$ exposure experiment in Fig. 6 (A), but there are also cells which exhibited peaks in calcium ion activity in tandem with magnetic field exposure. However, there were no cell responses which clearly synchronized in a $400 \mathrm{~Hz}, 500 \mathrm{mT}$ exposure experiment shown in Fig. 6 (B). No clear cell response was similarly observed in the $1-\mathrm{kHz}$ exposure experiments up to $400 \mathrm{mT}$.

Coil impedance due to inductance increased as frequency increased, making it difficult for current to flow. Therefore, the experiments shown in Fig. 6 generated a magnetic field at 750 $\mathrm{mT}$ for $200 \mathrm{~Hz}$ but at $500 \mathrm{mT}$ for $400 \mathrm{~Hz}$. It was thought that the magnetic field intensity for generating an induced electric field needed for a stimulus response in the cells could not be achieved, precluding the observation of a clear response. Meanwhile, the amount of estimated induction under these conditions and the external electric field applied to the cells during electric field stimulation due to more direct electrodes can both be estimated to be indirect applications without making contact with the cells as well as applying an external electric field which was similar and sufficient for a stimulus response [7]. Therefore, evaluation of the threshold by replacing the magnetic field stimulation with external electric field stimulation was possible.

We thus conducted experiments to obtain a threshold of cell response across a wide range of frequencies with stimulation experiments that used electrode-based external electric field applications.

\subsection{Characteristics of stimulus effects of time-vary- ing external electric fields}

\subsubsection{Exposure of time-varying external electric fields}

Electric field exposure to cells, in order to impart a locally large potential at the single cell level, was carried out by installing the uniquely manufactured U-shaped electrode (app. $1 \mathrm{~mm}$ gap, $3 \mathrm{~mm}$ length) located just above the cell on the bottom of culture dish (Fig. 7). The installation of electrodes in the vicinity of cells was operated using a micromanipulator (micro-support Co., Ltd., Japan), and the cells were installed between U-shaped electrodes. The electrode was connected to a signal generator (WF1943, NF Co, Japan), and experiments were conducted while changing the frequency and voltage. The frequency range was 10 $\mathrm{Hz}$ to $50 \mathrm{kHz}$, taking into account railway magnetic fields and the extrapolation band in the ICNIRP guidelines. The voltage ranged from about 0 to $10 \mathrm{~V}$, and the cells were observed while the voltage was changed in steps of minimum $0.1 \mathrm{~V}$. Responsiveness to external electric field stimulation was investigated using rat nerve cells. The stimulator/methods and observation methods for the calcium ion amounts are as shown in Section 4.1.1.

\subsubsection{Observation of stimulus response due to time- varying external electric field exposure}

A typical result where a voltage of 0 to $1.5 \mathrm{~V}$ at $3 \mathrm{kHz}$ is shown in Fig. 8. Approximately 10 cells (alternatively, clusters of cells) were selected for each cell (or clusters of several to ten cells) between the electrodes for external electric field stimulation, and fluorescence intensity was measured over time at each cell location. A typical cell (cell cluster) reaction of two cells is shown in Fig. 8: no cell response was observed with a $0.4 \mathrm{~V}$ application, but a cell response was confirmed with a $0.5 \mathrm{~V}$ application confirmed a cell response. In this case, the threshold value for nerve stimulation when a voltage was applied to nearby electrodes was determined to be $0.5 \mathrm{~V}$. Further increasing the voltage showed a higher fluorescence intensity as the voltage increased, with a rapidly increased response at $1.4 \mathrm{~V}$. The external electric field at 1.4 

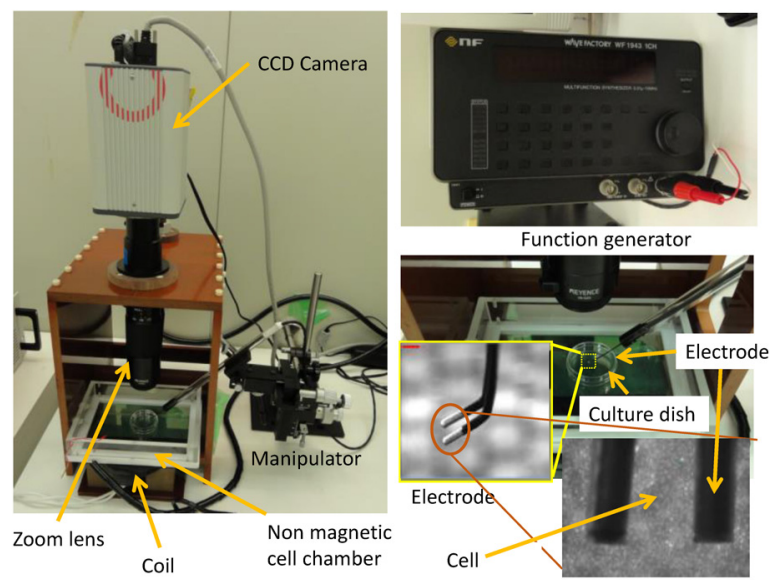

Fig. 7 Experimental setup for Electric field stimulation

$\mathrm{V}$ level can be considered the actual nerve stimulus response, but there are a limited number of studies which investigated the stimulus response characteristics at the single-cell level with external electric field applications at $0.1 \mathrm{~V}$ steps and across a wide range of frequencies from $10 \mathrm{~Hz}$ to $50 \mathrm{kHz}$, and no established criteria exist. Therefore, analyses were conducted with the threshold set to the external electric field where a cell response was first observed, as this was thought to be a safer evaluation.

Frequency characteristics ranging from $10 \mathrm{~Hz}$ to $50 \mathrm{kHz}$ were obtained for the stimulus response of nerve cells in the cerebral cortex of rat fetuses based on the results of the abovementioned tests. However, showing the estimated value of the electric field actually applied to cells requires the following:

- Measurement of the electric constant under experimental conditions

- Estimation of the potential between electrodes using simulations

- Estimation of the contribution of the electric double layer of electrodes placed near cells.

Therefore, for the stimulus response thresholds for external electric fields obtained in our wide-ranging experiments, the basic restrictions in the current ICNIRP guidelines and estimated thresholds for magnetic field or external electric field obtained in our study were plotted in Fig. 9. The threshold for the external electric field was determined by (1) measuring for each frequency the electric constant of

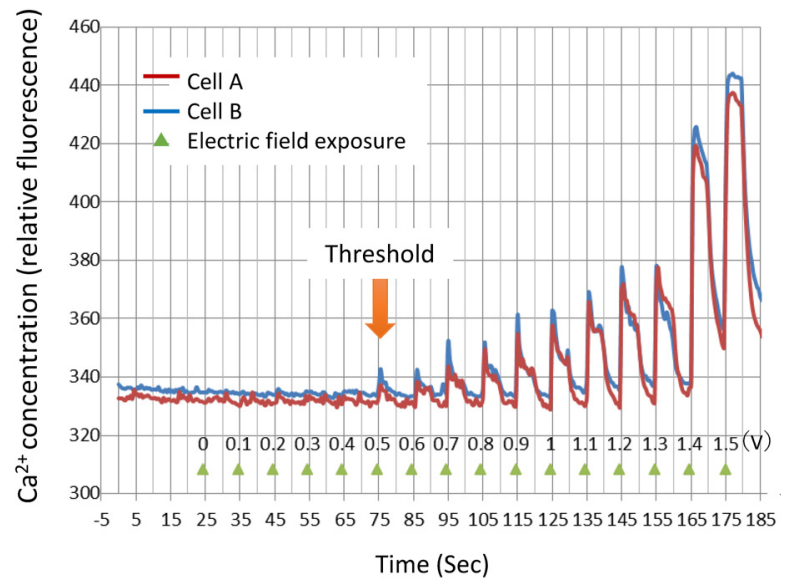

Fig. 8 Typical experimental results of external electric field stimulation of cultured rat nerve cells the culture under experimental conditions, (2) numerically modeling the electrode and culture container using finite element methods, and (3) simulating the spatial distribution of inter-electrode potential. Results showed that thresholds in our study were in line with the basic restrictions for occupational exposure in the current ICNIRP guidelines.

\subsubsection{Discussion}

The estimated values of the induced electric field in the stimulation threshold of the nerve cell response by external electric field obtained in this study, compared with the experimental values from existing volunteers. were relatively low.

Factors that may influence the accuracy of assessments of the estimated values include the extent of contribution of the electric double layer formed by the electrode surface during external electric field application, and the accuracy of the simulation parameters, which would need to be verified.

In addition, from a biological perspective, the cells used in this experiment formed cell clusters and two-dimensional random networks, which may have increased their sensitivity relative to peripheral nerves which are considered to be almost linear and one-dimensional. We plan to conduct further studies on these aspects in the future.

There is no prior study for the wide-ranging experiment results relating to nerve stimulus response obtained in this study, and it is thought that these will therefore serve as a solid foundation for supporting the basic restrictions in the current ICNIRP guidelines during future periodic reviews of the ICNIRP guidelines.

Furthermore, with regards to magnetic fields in railways, the data obtained from specific experiments on the extrapolated frequency bands which are currently not regulated for Japanese railways showed a high correlation with the current ICNIRP guidelines, so this may become the basis for showing that the possibility of the standards being more strict than the current ICNIRP guidelines is unlikely. Therefore, it seems that the measures based on the WHO recommendation and the current ICNIRP guidelines are appropriate for the protection of humans against the short-term effects of electromagnetic field exposure.

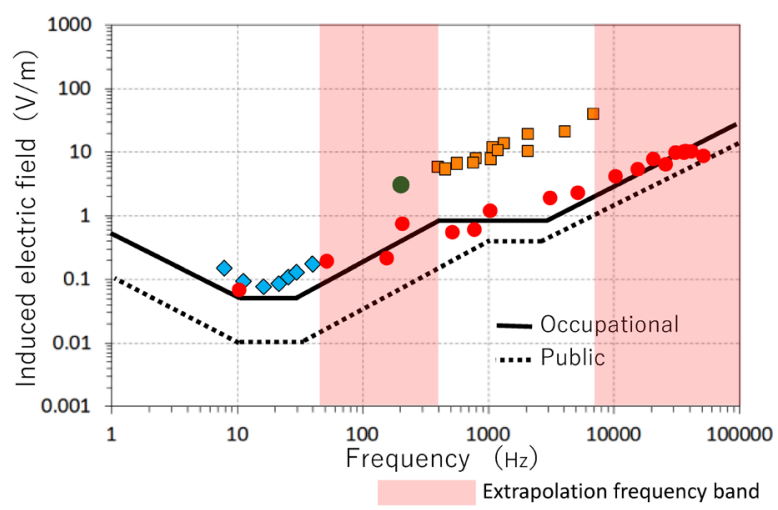

Fig. 9 Threshold of nerve stimulation in rat fetus' primary culture by external magnetic field / electric field plotted with the basic restriction of the ICNIRP guideline

Rat fetus' nerve cell (This study) $\bigcirc$ :electric field, $\bigcirc$ :magnetic field

Magnetophosphene

Peripheral nerve stimulation 


\section{Conclusion}

In response to the WHO's health risk assessment of electromagnetic fields below $100 \mathrm{kHz}$ and recommendations in 2007 and the subsequent release of the ICNIRP guidelines in 2010, corresponding regulations and standardization to protect people from the short-term effect of magnetic fields have been developed. However, considering the process of formulating the underlying ICNIRP guidelines, it was necessary to consider the validity of the guidelines for magnetic fields with a wide range of frequencies generated from railway systems.

In this study, we investigated characteristics of threshold of nerve stimulation by electric field instead of magnetic field using cultured neurons derived from the brain of rat fetuses. Our results showed that thresholds were in line with the basic restrictions for occupational exposure in the current ICNIRP guidelines. It will be a first solid evidence to support the guidelines over a wide frequency range including the extrapolation band. In order to gather more relevant evidence, future investigations will need to examine the characterization of the stimulus threshold in human nerve cells such as those derived from iPS cells.

In addition to such basic research, the Railway Technical Research Institute provides scientific evidence regarding magnetic fields in railways, such as the prediction methods of low frequency magnetic fields [8], the development of railway magnetic field measurement standards [9] and the development of materials for risk communication [10], and trend surveys of risk assessment based on the WHO's health risk assessment [11]. Various projects are underway to properly address this issue.

Magnetic fields generated in the railway system are mostly very low level. On the other hand, by properly recognizing the risks that can exist for such factors and raising awareness of this issue in society, we will continue to study to improve the perception of safety and security of the railways, to reinforce public trust.

\section{Acknowledgement}

We would like to express our appreciation to Prof. Keiji Wada, , Tokyo Metropolitan University for his contribution in the development of the magnetic field generator for cell exposure.

\section{References}

[1] Wertheimer, N. and E. Leeper: "Electrical wiring configurations and childhood cancer," Am. J. Epidemiol., 109, pp. 273-284, 1979.

[2] International Commission on Non-Ionizing Radiation Protection, "Guidelines for Limiting Exposure to Time-Varying Electric and Magnetic Fields (1 Hz - 100 kHz)," Health Physics, Vol. 99, 818-836 (2010).

[3] WHO: Environmental Health Criteria 238: Extremely Low Frequency Fields (2007).

[4] Reilly JP, "Neuroelectric mechanisms applied to low frequency electric and magnetic field exposure guidelines-part I: sinusoidal waveforms," Health Phys, 83:341-355, 2002.

[5] Saunders RD, Jefferys JG, "A neurobiological basis for ELF guidelines,” Health Phys, 92:596-603, 2007.

[6] Wada, K., Hayashi, S., Suzuki, Y., Ikehata, M., Yoshie, S., Saito, A., Nakasono, S., "Design and Implementation of Multi-Frequency Magnetic Field Generator Producing Sinusoidal Current Waveform for Biological Researches," 18th European Conference on Power Electronics and Applications, 2016.

[7] Ikehata, M., Wada, K., Makino, K., Suzuki, Y., Saito, A., Yoshie, S., Nakasono S., "Real-time detection of neuronal activity induced by time-varying magnetic field or electric field exposure in in vitro," BioEM 2018, Piran, Slovenia, 2018.

[8] Kato Y., Hasegawa H., "Measurement and Prediction Method Related to the Low-Frequency Magnetic Field on Railway Vehicles," RTRI Report, Vol.28, No.9, pp. 29-34, 2014 (in Japanese).

[9] IEC 62597 ed1.0: "Measurement procedures of magnetic field levels generated by electronic and electrical apparatus in the railway environment with respect to human expo-sure," 2019.

[10]Ikehata, M., Kato, Y., Morita, G., Nakamura, K., Sasakawa, T., "EMF issue in railway system -evaluation and communication tool," IP_07_6," 12th World Congress on Railway Research, Tokyo, Japan, 2019.

[11] Ikehata, M., Nakasono, M., Shigemitsu, T., Miyagi, H., "Trends in health risk assessment related to extremely low frequency EMF," IEEJ Annual Meeting, S4-3, (in Japanese) 2017.

\section{Authors}

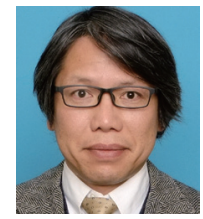

Masateru IKEHATA, Ph.D.

Senior Chief Researcher, Head of

Biotechnology Laboratory, Human Science

Division

Research Areas: Genotoxicity,

Bioelectromagnetics, Occupational Hygienes

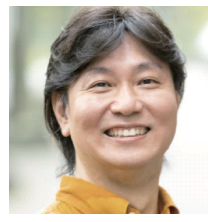

Yukihisa SUZUKI, Ph.D.

Professor, Department of Electrical

Engineering and Computer Science,

Graduate School of Systems Design, Tokyo

Metropolitan University Research Areas:

Bioelectromagnetics, Electromagnetic

Compatibility, High Performance Computing

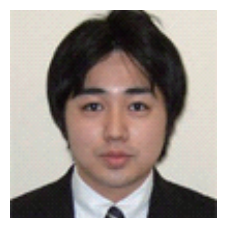

Atsushi SAITO, Ph.D

Research Scientist, Biological Environment Sector, Environmental Science Research Laboratory, Central Research Institute of Electric Power Industry (CRIEPI)

Research Areas: Bioelectromagnetics, Neurophysiology, Cell Biology

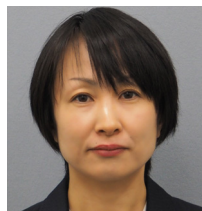

Sachiko YOSHIE, Ph.D.

Assistant Senior Researcher, Biotechnology

Laboratory, Human Science Division

Research Areas: Molecular Biology,

Microbiology, Bioelectromagnetics 